\title{
EVOLUTION RECENTE DE LA MORPHOLOGIE DE DELTA DE FLOT ET SON EFFET SUR LA DYNAMIQUE HYDRO-SEDIMENTAIRE DE LA LAGUNE DE OUALIDIA (CÔTE ATLANTIQUE, MAROC): APPROCHE PAR PHOTOGRAPHIE AÉRIENNE
}

\author{
El Khalidi Khalid ${ }^{(1)}$, BendahHou ZouraraH(1) \& Ahmed AajJane(1)
}

Résumé

Abstract

\begin{abstract}
L'analyse diachronique par photo-interprétation de l'évolution du delta de flot de la lagune de Oualidia révèle mobilité morpho-sédimentaire de ce corps sableux sur 52 ans marquée par une expansion continue de 1954 à 2006. Cette expansion est due à la présence de sables provenant de l'érosion de la côte adjacente et des stocks sédimentaires de la plate forme continentale mobilisés grâce aux courants induits par la houle et les courants de marée qui sont les principaux courants intra-lagunaires. La lagune de Oualidia est une lagune à flux dominée par les courants de flot qui sont plus importants que ceux du flot. Le dynamisme et la rapide évolution morphologique du delta de flot de la lagune de Oualidia, sous l'influence de forçages hydrodynamiques, ne sont pas sans conséquences écologiques et économiques notamment sur le confinement et la salubrité du milieu.
\end{abstract}

Mots-clés: Analyse diachronique; Évolution morpho-sédimentaire; Delta de flot; Lagune de Oualidia.

\begin{abstract}
Recent changes of a flood-tide delta morphology and effects on the hydro-sedimentary dynamics of the Oualidia lagoon (Atlantic Coast, Morocco): an approach by aerial photography analysis The diachronic analysis by photo interpretation of the flood-tidal delta of the Oualidia lagoon reveals morphosedimentary mobility of this sandy accumulation along 52 years marked by a continuous expansion between 1954 and 2006. This expansion is possible due to the presence of sand eroded from the adjacent coast and to the stocks of the continental shelf sediments mobilized by currents induced by waves and tidal currents that are predominant inside the lagoon. The Oualidia lagoon is dominated by flood currents that are higher than ebb currents. The dynamic and the morphological evolution of the flood-tidal delta of the Oualidia lagoon under the influence of hydrodynamic forcing, induce ecological and economic consequences with implications in confinement and environmental health.
\end{abstract}

Key-words: Diachronic analysis; Morpho-sedimentary evolution; Flood-tidal delta; Oualidia Lagoon.

\section{INTRODUCTION}

Le Maroc compte parmi les pays riches en zones humides (estuaires, lagunes, lacs, dayas, cours d'eau...). Il est doté de quatre principales lagunes sur une côte marine d'environ $3600 \mathrm{~km}$.

Parmi les trois lagunes sur la côté atlantique marocaine, la lagune de Oualidia est connue par son importance ornithologique. Elle a été classée entant que site d'intérêt biologique et écologique (S.I.B.E), à travers le Plan directeur des aires protégées, et come site RAMSAR depuis 2005. Ce site, de valeur internationale, constitue une escale migratoire et un refuge hivernal apprécié par les différentes espèces d'oiseaux d'eau parmi lesquels les limicoles viennent en tête de liste avec 26 espèces suivi par les anatidés et les laridés (EL Hamoumi 2000).

D'après Paskoff (2001), les zones côtières souffrent actuellement d'une élévation du niveau de la mer qui devrait accentuer l'érosion des côtes et, éventuellement, la déclencher là où elle n'apparaît pas encore. Selon le groupe intergouvernemental des études de climat (GIEC 2007), cette élévation de niveau de la mer est principalement due au réchauffement du climat. Seules résisteront à l'érosion, les plages suffisamment alimentées en sédiments pour contrecarrer les effets d'une élévation du niveau de la mer (LEATHERMAN 1990).

Les matériaux d'érosion des plages sont dispersés par les vagues et les courants et ils peuvent soit

(1) Laboratoire de Géosciences Marines et Sciences du Sol, Unité associé au CNRST (URAC 45). Université Chouaib Doukkali, B.P. 20, 24000 El Jadida, Morocco. E-mail: elkhalidi_khalid@yahoo.fr 
transportés par la dérive littorale pour être remanier sur les côtes adjacent, soit rejoindre les fonds marins.

Les deltas de flot des lagunes, sont de véritables réservoirs de sable souvent utilisés dans les opérations d'aménagements côtiers, et leur volume peut atteindre celui des îles barrières (FITZGERALD et HAYES 1980). Des variations des conditions hydrodynamiques de ces systèmes sableux peuvent engendrer des variations du transport sédimentaire, qui vont à leur tour agir sur le volume de sable transféré à la côte aval, conditionnant l'accrétion ou l'érosion de ses plages. La migration des passes peuvent également avoir des effets néfastes sur les côtes adjacentes.

Qu'une lagune puisse demeurer ouverte est d'un grand intérêt, et ce, pour plusieurs raisons. Les passes des lagunes sont tout d'abord l'unique moyen de régénérer l'eau présente dans la lagune et de lui fournir une eau côtière riche en oxygène. La qualité de l'eau du système lagunaire et les éventuelles activités d'aquaculture en dépendent.

De nombreux travaux ont été réalisés pour caractériser la lagune de Oualidia et son écosystème (Beaubrun 1976; Carruesco 1989; Orbi et al. 1998; RHARBI et al. 2001; HILMI et al. 2005; KoUTITONSKY et al. 2006). Depuis quelques années, la qualité des eaux de la lagune se détériore, probablement suite à un confinement des eaux en certains endroits en amont dans la lagune (KOUTITONSKY et al. 2006).

L'objectif du présent travail est d'évaluer l'évolution morpho-sédimentaire du delta de flot de la lagune de Oualidia entre 1954 et 2006, à travers un suivi diachronique par l'analyse des photographies aériennes, et de discuter la nature et l'origine de ces sédiments.

\section{Presentation du Site D’etude}

Située sur le littoral atlantique à $76 \mathrm{~km}$ au sud d'El Jadida, la lagune de Oualidia (32 $44^{\circ} 42^{\prime \prime} \mathrm{N}$ et 09 02' 50” W) (Fig. 1), petite station balnéaire, est l'un des sites littoraux au Maroc où se développent plusieurs activités à caractère socio-économique dont l'ostréiculture. Elle s'inscrit dans un domaine de 7 $\mathrm{km}$ de long sur 0,4 à $0,5 \mathrm{~km}$ de large et sa superficie totale est évaluée à $3 \mathrm{~km}^{2}$ (BEAUBRUN 1976). Quant au climat, la région d'étude est connue par un climat de type méditerranéen semi aride mais la bande côtière subit des influences atlantiques.

De l'aval vers l'amont de la lagune, trois unités morphologiques y sont distinguées (CARRUESCO 1989; ZOURARAH 2002) (Fig. 1):
- une passe principale de $150 \mathrm{~m}$ de large environ, permanente et active toute l'année; et une deuxième passe adjacente, de $50 \mathrm{~m}$ de large, active uniquement en période des marées de vives-eaux: ces deux passes assurent à la lagune une communication continue avec l'Océan Atlantique;

- un chenal principal dont la profondeur maximale ne dépasse pas 5-6 m, les chenaux secondaires (de profondeur maximale 1 à $1,5 \mathrm{~m}$ ), les zones intertidales et les schorres envahis par une végétation halophyte;

- une digue artificielle séparant l'amont de la lagune des marais salants.

La lagune de Oualidia est mise en place lors de la transgression mellahienne (Holocène moyen) au sein de la dépression de l'Oulja; cette dernière délimite le domaine océanique du domaine continental, en l'occurrence le bassin endoréique côtier du Sahel, prolongé à l'Est par la plaine des Doukkala (ZOURARAH 2002). La lagune est alimentée essentiellement par des eaux de mer et par des quantités nettement moins importantes des eaux phréatiques. La dynamique combinée de ces composantes hydrologiques génère une mosaïque plus ou moins complexe de sédiments, de mécanismes géochimiques et de communautés végétales (KOUTITONSKY et al. 2006).

La marée est de type semi diurne et la côte est mésotidale. La vitesse moyenne des courants, mesurée au niveau de la passe en 2002 est assez élevée (0,5 à $1 \mathrm{~m} / \mathrm{s})$ (GEME et al. 2003). Les données de houle montrent que l'évolution du littoral de la lagune de Oualidia se fait sous l'influence combinée des houles dominantes des secteurs NNW et NW (78\%) avec des houles secondaires de direction $\mathrm{N}$, WNW et W (CHAIBI 2003).

Il a été remarqué récemment que la qualité des eaux de la lagune de Oualidia a diminué, probablement en raison des changements de l'hydrodynamique à l'embouchure où se développe le delta de flot (KoUTITONSKY et al. 2006).

\section{Materiel et Methodes}

L'analyse diachronique de l'évolution de la zone côtière de la lagune de Oualidia repose sur la comparaison des photos aériennes prises à trois différentes missions: 1954 (1/10.000), 1984 (1/20.000) et $2006(1 / 20.000)$. Comme document de référence nous avons utilisé la carte topographique (échelle: 1/25.000, source: L'Agence Nationale de la Conservation Foncière, du Cadastre et de la Cartographie, Maroc). Les photos aériennes utilisées présentent des déformations (DURAND 2000; ARIAS 2003; DEHOUCK 


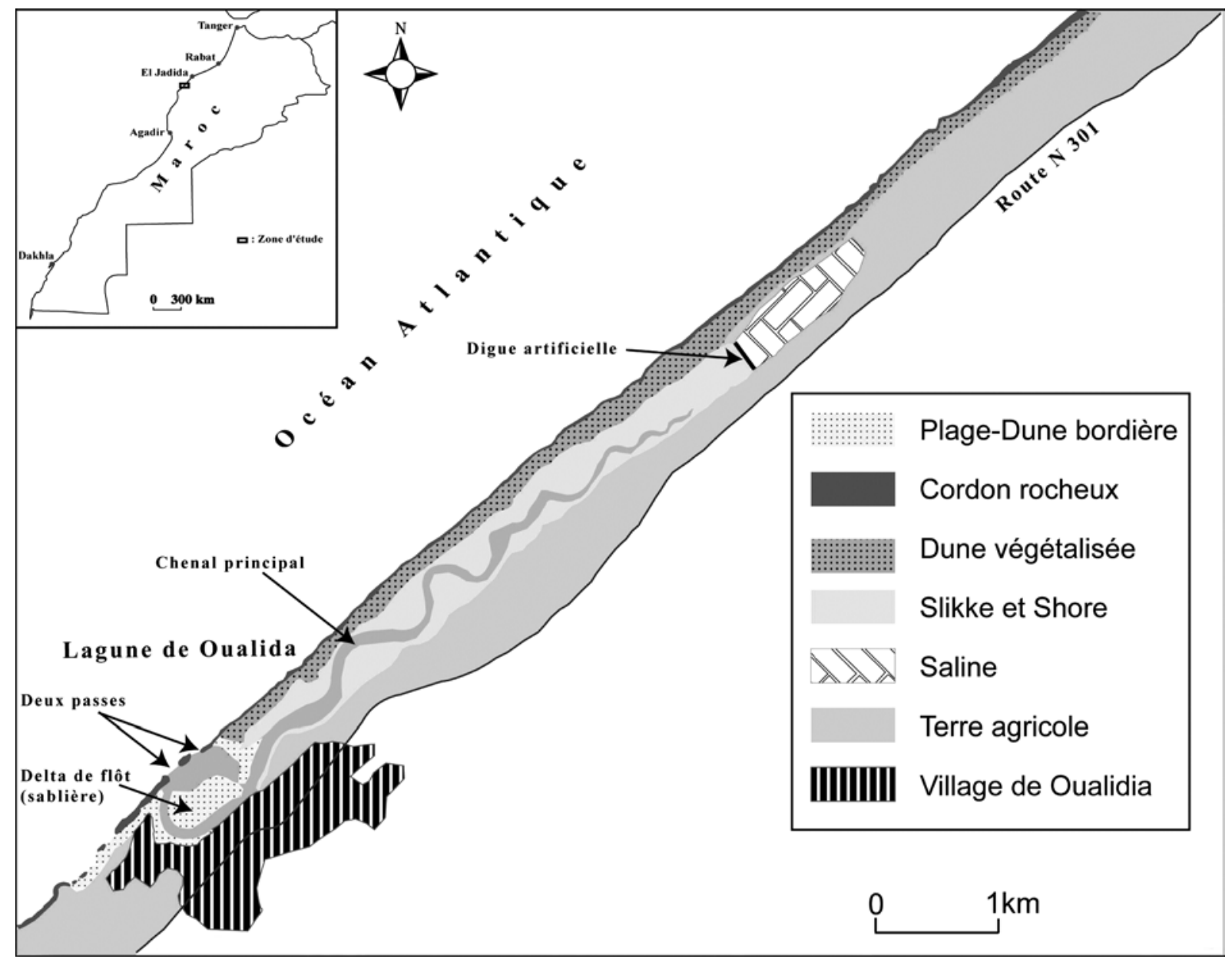

Fig. 1. Carte de localisation et d'occupation du sol de la lagune de Oualidia (Côte atlantique marocaine).

Fig. 1. Location map and land use of the Oualidia lagoon (Moroccan Atlantic coast).

2006), ne permettant pas une superposition directe avec la carte topographique. Il est donc nécessaire de corriger ses erreurs pour obtenir des images dont l'échelle est constante en tout point.

La correction et le géoréférencement des documents a été réalisés grâce aux logiciels TNTmips (MicroImages) et Arcgis. En premier lieu, nous avons commencé le traitement par le géoréférencement de la carte topographique. Nous avons choisi le Datum de Merchich et la projection Lambert Conformal Conic Maroc Nord. Le géoréférencement des photos aériennes est réalisé à partir de la carte topographique précédemment référencée, ce qui implique la saisie de points homologues (amers) entre les deux images (Dolan et al. 1980; Crowell et al. 1991 GAILlot \& Chaverot 2001; Menie Ovono 2010). Ces amers doivent être répartis sur la totalité de la photographie pour obtenir un résultat cohérent.

Pour corriger au mieux les défauts liés aux distorsions internes des photos aériennes, nous avons utilisé le "modèle Affine" du logiciel TNTmips.
Lors du processus de rectification géométrique, la nouvelle image discrétisé peut avoir un nombre de lignes et de colonnes différents de celui de l'image de départ. Le processus de ré-échantillonnage que nous avons utilisé (par plus proche voisin) permet de déterminer la valeur numérique à placer dans la nouvelle localisation de chaque pixel de l'image de sortie corrigée. La dernière étape consiste à superposer les photos aériennes et de digitaliser le contour du delta de flot afin de quantifier sa superficie à différentes dates.

\section{Resultats et Discussion}

L'étude diachronique sur 52 ans menée pour le delta de flot de la lagune de Oualidia (Fig. 2), permet de dégager les modifications suivantes:

- Le delta a connu une expansion continue de 1954 à 2006. Sa superficie est passée de $360.10^{3} \mathrm{~m}^{2}$ en 1954 à $432.10^{3} \mathrm{~m}^{2}$ en 1984 , ce 
qui représente une augmentation de $20 \%$ durant 30 ans. Et entre 1984 et 2006, la superficie est passée de $432.10^{3} \mathrm{~m}^{2}$ à $444.10^{3}$ (environ $3 \%$ pendant les 22 années plus récentes);

- Pendant l'ensemble de la duré étudiée - de 1954 à 2006 (52 ans) - le delta a connu une expansion de l'ordre de $84.10^{3} \mathrm{~m}^{2}$.

Les sources de sédiments qui alimentent ce delta de flot sont le résultat combiné de deux principaux éléments. D'un côté, la morphologie de part et d'autre de l'embouchure de la lagune de Oualidia est dominée par des systèmes de cordons dunaires gréseux pliocènes et quaternaires; ces formes et matériaux littorales sont attaquées par la mer et donnent naissance aux sables locales qui peuvent être remobilisés par les vagues et les courants à l'intérieure de la lagune. D'un autre côté, un stock important de sable est mis en place lors de la dernière régression (JAAIDI 1981) sur le plateau continental au large de Oualidia (CIRAC et al. 1979; El Foughali 1982; JAAÏDI \& CIRAC 1987). Ce stock constitue un réservoir potentiel des sédiments pour le delta de flot de la lagune. Ce sable peut être mobilisé lors des tempêtes que connait la région durant l'hiver. La dérive littorale permet de remanier ces matériaux aux alentours de la passe de la lagune.

D'autre part, à partir des résultats obtenus, on s'aperçoit qu'au cours de la période étudiée, le chenal principal de la lagune était subdivisé (1954 et 1984) en deux chenaux de part et d'autre de la sablière, avant que le chenal situé au Nord de celle-ci se soit rétréci (en 2006) (Fig. 2). Ceci est dû d'une part, au déferlement des vagues qui subit un amortissement par le sable du delta de flot qui se développe et, d'autre part, et selon des mesures réalisées en 2002 pour étudier les courants de marée (GEME et al. 2003), cette lagune est dominée par le flux et les courants à l'intérieur de la lagune sont liés à la

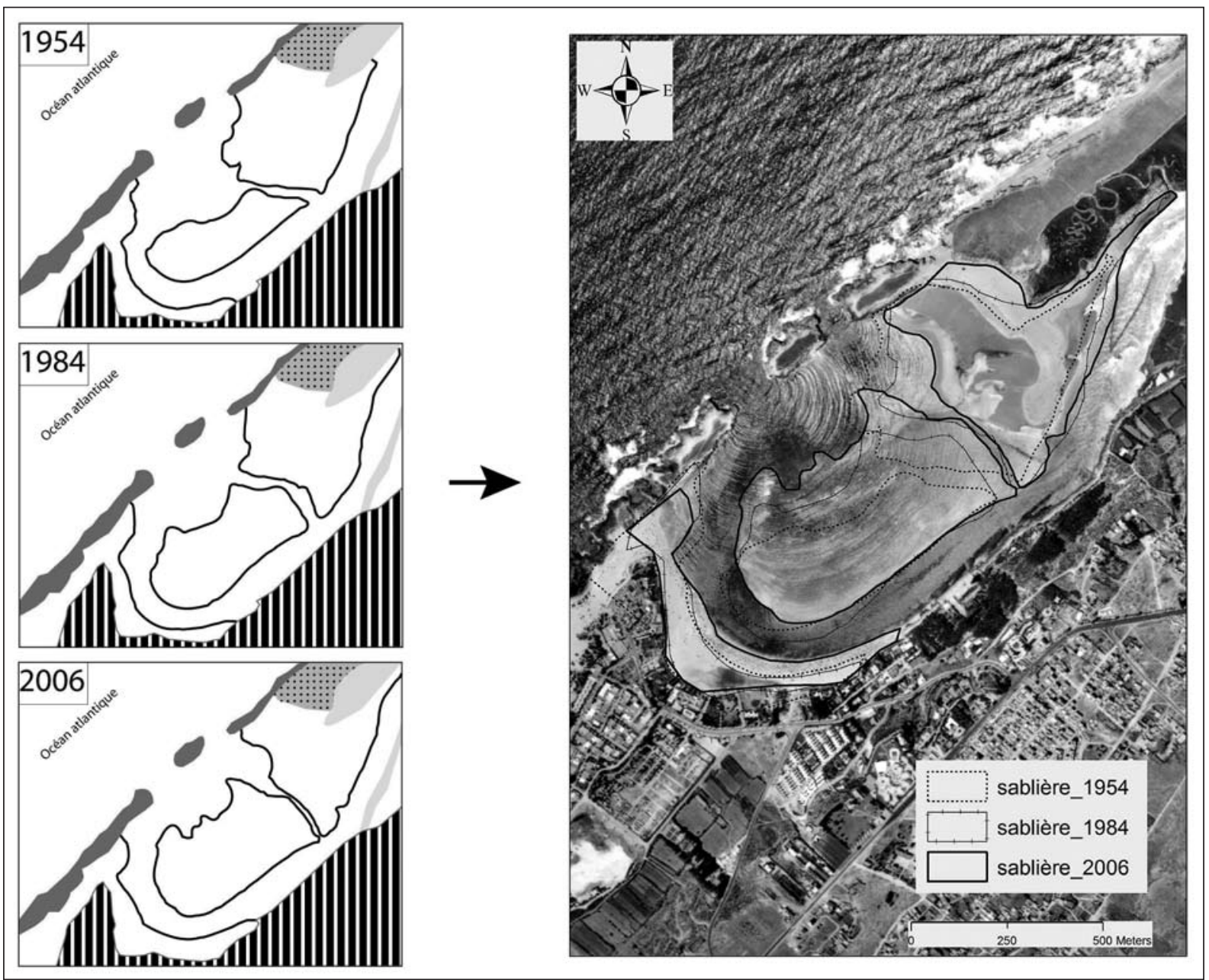

Fig. 2. Evolution diachronique du delta de marée de la lagune de Oualidia entre 1954 et 2006 (A droite, photo aérienne prise en 2006).

Fig. 2. Diachronic evolution of the tidal delta of the lagoon of Oualidia between 1954 and 2006 (At right, aerial photograph in 2006). 
forme de delta. D'après ces mesures, l'amplitude de la marée à l'embouchure est d'environ $1,5 \mathrm{~m}$ et la profondeur moyenne dans la zone des chenaux en amont est de l'ordre de 1,5 m. La direction des courants subit donc des changements dus à l'avancement de la sablière vers les passes de la lagune. Le sens du transport des sédiments à l'intérieur de la lagune est alors affecté ce qui pourrait permettre l'accroissement de la sablière plutôt que la dispersion de ces sédiments. Au cours du jusant, il sera alors plus difficile au sable de retourner vers la mer.

Ce genre de phénomène d'ensablement des passes a été aussi observé par El Khalidi et al. (2007) au niveau de la passe de la lagune de Sidi Moussa à 37 $\mathrm{km}$ au Nord de Oualidia.

\section{Conclusion}

L'analyse diachronique de delta de flot de la lagune de Oualidia, par la superposition des photographies aériennes de différentes missions durant 52 ans, nous a permis de mettre en évidence des changements morphologiques et un accroissement de la sablière ce qui a affecté la dynamique des sédiments au niveau de cette lagune. L'étude a permis également de discuter les processus hydrodynamiques qui contrôlent cette portion du littoral marocain.

Des investigations supplémentaires permettront de montrer que ces variations observées sont à l'origine de perturbation du fonctionnement de l'écosystème de ce site d'intérêt biologique et écologique où d'autres facteurs peuvent également intervenir.

Cette étude et d'autres antérieures sur la lagune de Oualidia relatent une prise de conscience relativement précoce des baisses de valeur que subissent cette lagune, sous l'effet des forçages naturels et du phénomène d'ensablement de sa passe. Pourtant, la volonté de protéger ces milieux est continuellement manifestée, mais elle se heurte à plusieurs obstacles: le partage de la gestion des lagunes entre plusieurs secteurs et la faiblesse du cadre juridique en matière de conservation des milieux aquatiques au Maroc.

Pour mieux cerner ces dysfonctionnements, des solutions doivent être apportées à travers des programmes de suivi, basés sur une connaissance approfondie du fonctionnement des milieux lagunaires et des enjeux de leur conservation.

\section{REMERCIEMENTS}

Ce travail a été réalisé grâce aux différents soutiens: Projet CNR-CNRST SH01, PAI MA/07/179, et du programme LagMar du Réseau REMER au Maroc.

\section{BIBLIOGRAPHIE}

ARIAS, C.A. 2003. Spatio-temporal analysis of Texas shoreline changes using Gis technique. Thèse université Texas A\&M, $79 \mathrm{p}$.

BeAubrun, P.C. 1976. Les huîtres au Maroc et l'ostréïculture dans la lagune de Oualidia. Bull. Inst. Pêches Maritimes 22: 13-143.

CARRuesco, CH. 1989. Genèse et évolution à l'holocène de trois lagunes de la façade atlantique: Moulay Bousselham, Oualidia (Maroc) et Arcachon (France). Bordeaux: Univ. Bordeaux I (Thèse Doctorat d'Etat).

CHaibI, M. 2003. Dynamique sédimentaire et morphogenese actuelle du littoral d'El Jadida (Maroc). Aix-Marseille: Université Aix-Marseille (Thèse de Doctorat Es-sciences).

Cirac, P.; Faugeres, J.C. \& Gayet, J. 1979. Résultats préliminaires d'une reconnaissance sédimentaire du plateau atlantique marocain. Bull. Inst. Géol. Bassin d'Aquitaine 25: 69-81.

Crowell, M.; Leatherman, S.P. \& Buckley, M.K. 1991. Historical shoreline change: Error Analysis and Mapping Accuracy. Journal of Coastal Research 7 (3): 839-852.

DeHoucK, A. 2006. Morphodynamique des plages sableuses de la mer d'Iroise (Finistère). Université de Bretagne Occidentale (Thèse de doctorat), 262p.

Dolan, R.; HAYden, B.P.; MAY, P. \& MAY, S. 1980. The reliability of shoreline change measurements from aerial photographs. Shore and Beach 48: 22-29.

DurAND, P. 2000. Approche méthodologique pour l'analyse de l'évolution des littoraux sableux par photo-interprétation, Exemple des plages situées entre les embouchures de l'Aube et d'Hérault (Languedoc, France). Photo-interprétation 18: 1-2, 3-18.

El Foughali, A. 1982. Analyse morphostructurale du plateau continental atlantique marocain de Tanger à cap Cantin, Maroc. Bordeaux: Univ. Bordeaux I n. ${ }^{\circ} 1824$ (Thése 3 ème Cycle).

El Khalidi, K.; Zourarah, B.; Chaibi, M.; MaAnan, M. \& LEONE, F. 2007. Morphological and diachronic stady of the lagoon of Sidi Moussa, Morocco. Terra et aqua 106: 3-9.

FitzGerald, D.M. \& Hayes, M.O. 1980. Tidal inlet effects on barrier island management. Coastal zone. Amer. Soc. Of Civil Engrs: 2355-2379.

EL Hamoumi, R. 2000. L'avifaune aquatique du complexe lagunaire de Sidi Moussa-Walidia (Maroc). Fac. Sci. Casablanca (Thèse de doctorat d'état es-Sciences Biologie), 241 pp.

Gaillot, S. \& Chaverot, S. 2001. Méthode d'étude des littoraux à faible évolution. Cas du delta du Golo (Corse) et du littoral du Touquet (Pas-de-Calais) en France. Géomorphologie: relief, processus, environnement 7 (1): 47-54.

GEME, Hydro-M. \& IN VIVo 2003. Diagnostic et études du confinement de la lagune de Oualidia - Phase 2 - Campagne de mesures. Rapport définitif. Royaume du Maroc: Direction Provinciale de l'Equipement d'El Jadida, Ministère de l'Equipement et du Transport, 60p.

GIEC 2007. Bilan 2007 des changements climatiques. Contribution des Groupes de travail I, II et III au quatrième Rapport d'évaluation du Groupe d'experts intergouvernemental sur l'évolution du climat [Équipe de rédaction principale, $\mathrm{Pa}-$ chauri, R.K. \& Reisinger, A.]. Genève: GIEC.

Hilmi, K.; OrBI, A.; LAKHDAR, J.I. \& SARF, F. 2005. Etude courantologique de la lagune de Oualidia (Maroc) en automne. Bulletin de l'Institut Scientifique. Section Sciences de la Vie 26-27: 67-71.

Koutitonsky, V.G.; OuABI, M. \& IBRAhimi, I. 2006. L'étude du comportement hydrosédimentaire du système lagunaire Ouali- 
dia par la modélisation mathématique. Phase 1: Synthèse des données et simulations de la réfraction des houles. Royaume du Maroc: Direction des Ports et du Domaine Public Maritime, Ministère de l'Équipement et du Transport.

LEATHERMAN, S.P. 1990. Modelling shote response to sea-level rise on sedimentary coasts. Progress in physical geography 14 (4): 447-467.

Menie Ovono, Z. 2010. Évolution de la flèche Mandji de l'Holocène à l'actuel. Analyse et cartographie du risque côtier. Nantes: Université de Nantes (Thèse de doctorat).

JAAIDI, E.B. 1981. Les environnements sédimentaires actuels et pléistocènes du plateau continental atlantique marocain entre Larache et Agadir. Bordeaux: Univ. Bordeaux I (Thèse 3ème cycle).

JAAIDI, E.B. \& CIRAC, P. 1987. La couverture sédimentaire meuble du plateau continental atlantique marocain entre Larache et Agadir. Bull. Inst. Géol. Bassin d'Aquitaine 42: 33-51.
PASKOFF, R. 2001. L'élévation du niveau de la mer et les espaces côtiers. Le mythe et la réalité. Collection "Propos". Paris: Institut Océanographique.

ORBI, A.; HILMI K.; LARISSI, J.; ZIDANE, H.; ZIZAH, S.; El MoUSSAOUI, N.; LAKHDAR, J.I. \& SARF, F. 1998. Hydrologie et hydrodynamique des côtes marocaines: milieux paraliques et zones côtières. Lisbonne: Commissariat général Expo'98.

Rharbi, N.; RAMdANi, M.; Berraho, A.B. \& LAKhdAR, J.I. 2001. Caractéristiques hydrologiques et écologiques de la lagune de Oualidia: milieu paralique de la côte atlantique marocaine. Marine Life 11/1-2: 3-9.

ZOURARAH, B. 2002. Les processus côtiers actuels et leur impact sur l'environnement littoral des Doukkala (côte atlantique marocaine): Approche hydrodynamique, Morphologique, Sédimentologique et Géochimique. El Jadida: Université Chouaïb Doukkali (Thèse de Doctorat d'état). 